\title{
Actividad hipoglucemiante de Bouvardia terniflora, Brickellia veronicaefolia y Parmentiera edulis
}

\author{
Rosa Martha Pérez-Gutiérrez, Dra. en $C{ }_{.1}{ }^{(1)} C$ uauhtémoc Pérez-González, $M$. en $C .{ }^{\left({ }^{(2)}\right.}$
}

Miguel Angel Zavala-Sánchez, M. en C., ${ }^{(2)}$ Salud Pérez-Gutiérrez, Dra. en C. ${ }^{(2)}$

\begin{abstract}
Pérez-Gutiérrez RM, Pérez-González $C$, Zavala-Sánchez MA, Pérez-Gutiérrez $S$. Actividad hipoglucemiante de Bouvardia terniflora, Brickellia veronicaefolia, y Parmentiera edulis. Salud Publica Mex 1998;40:354-358.
\end{abstract}

\section{Resumen}

Objetivo. Evaluar la actividad hipoglucemiante de los extractos de hexano, cloroformo y metanol de Brickellia veronicaefolia, Bouvardia terniflora y Parmentiera edulis. Material y métodos. Se probaron los extractos de las plantas (100, 200 y $300 \mathrm{mg} / \mathrm{kg}$, vía intraperitoneal) en ratones normoglucémicos y con diabetes inducida con aloxana. Resultados. La administración de $300 \mathrm{mg} / \mathrm{kg}$ de los extractos clorofórmicos de P. edulis, $B$. terniflora y hexánico de B. veronicaefolia en ratones diabéticos disminuye el nivel de glucosa sanguínea en $43.75,58.56$ y $72.13 \%$, respectivamente. Estos extractos (300 mg/kg), administrados en ratones no rmo glucémicos, reducen la glucosa sanguínea en $29.61,33.42$ y $39.84 \%$, respectivamente. Conclusiones. Con este estudio se confirma la actividad hipoglucemiante de estas plantas usadas en la medicina tradicional para el tratamiento de la diabetes.

Palabras clave: hipoglicemia; plantas medicinales/Brickellia veronicaefolia, Bouvardia terniflora, Parmentiera edulis
Pérez-Gutiérrez RM, Pérez-González $C$, Zavala-Sánchez MA, Pérez-Gutiérrez $S$. Hypoglycemic activity of Bouvardia terniflora, Brickellia veronicaefolia, and Parmentiera edulis.

Salud Publica Mex 1998;40:354-358.

\section{Abstract}

Objective. To evaluate the hypoglycemic activity of the hexane, chlo roform and methanol extracts of Bouvardia terniflora, Brickelia veronicaefolia and Parmentiera edulis. Material and methods $\mathrm{N}$ ormal and alloxan-induced diabetic mice were administered these plant extracts (intraperitoneal 100, 200 and $300 \mathrm{mg} / \mathrm{kg}$ ). Results. The administration of 300 $\mathrm{mg} / \mathrm{kg}$ of chloroform extracts from P. edulis and B. terniflora and hexane from B. veronicaefolia to diabetic mice decreased the blood glucose levels in $43.75,58.56$ and $72.13 \%$, respectively. These extracts administered to normal mice reduced blood glucose levels in 29.61, 33.42 and $39.84 \%$, respectively. Conclusions. The hypoglycemic effect of these plant extracts used in traditional medicine for diabetes treatment is confirmed.

Key words: hypoglycemic; plants, medicinal/Brickellia veronicaefolia, Bouvardia terniflora, Parmentiera edulis
L a Bouvardia terniflora (Cav) Schl. es una planta L comúnmente conocida como "trompetilla" o "expatli“ que pertenece a la familia de las rubiáceas. Es una especie que crece en climas secos, semisecos y templados, desde el nivel del mar hasta los $3000 \mathrm{~m}$ de altura. En los estados de Puebla y Morelos se utiliza la cocción de esta planta como antídoto para el veneno de víbora, abeja, alacrán, araña, ciempiés y hormiga, así como para combatir la tos, la disentería y los dolores de estómago y de cabeza; asimismo, se recomienda ampliamente como analgésico de aplicación tópica. En la práctica de la medicina tradicional, el extracto acuoso de sus hojas se emplea para el tratamiento de la diabetes. Bates y colaboradores aislaron el 6-O-me-

(1) Laboratorio de Investigación de Productos N aturales, Escuela Superior de Ingeniería Q uímica e Industrias Extractivas, Instituto Politécnico $N$ acional, México.

(2) Departamento de Sistemas Biológicos, Universidad Autónoma Metropolitana-Xochimilco, México.

Fecha de recibido: 29 de julio de 1997 - Fecha de aprobado: 11 de mayo de 1998 Solicitud de sobretiros: Dra. Rosa Martha Pérez. Punto Fijo 16, colonia Torres de Lindavista, 07750 México, D.F., México. 
tilbouvardin de la B. terniflora, que presenta propiedades antitumorales y citotóxicas. ${ }^{1}$

La Brickellia veronicaefolia (HBK) Gray, conocida comúnmente como "hierba dorada", es una planta que pertenece a la familia de las compuestas. En la medicina tradicional mexicana se emplea en el tratamiento de la diabetes mellitus, gastroenteritis, afecciones del estómago y algunos problemas de diarrea; en Michoacán se le atribuyen también propiedades vermífugas. En investigaciones anteriores se demostró la propiedad antidiarreica del extracto acuoso de esta planta en animales de experimentación. ${ }^{2}$ La B. veronicaefolia es propia de los climas semicálidos y templados entre los 1800 y $2800 \mathrm{~m}$ de altura.

La Parmentiera edulis D.C., conocida como "cuajilote", pertenece a la familia de las bignonáceas; esta planta se encuentra en los climas cálidos, semicálidos y templados desde los $2 \mathrm{~m}$ hasta los $2240 \mathrm{~m}$ de altura. El té del fruto, la raíz y la corteza se emplea para combatir el dolor de riñones. Su fruta se utiliza popularmente en el tratamiento de la diabetes mellitus en diversas regiones de los estados de Veracruz, Tabasco y Chiapas. En Veracruz y Quintana Roo se come el fruto sancochado y se utiliza para padecimientos tan diversos como el dolor de cabeza, los cálculos biliares, la sordera y la diarrea.

En este trabajo se determinó la actividad hipoglucemiante de los extractos de hexano cloroformo y metanol de las hojas de B. terniflora y B. veronicaefolia, así como de los frutos de P. edulis, usando ratones normoglucémicos e hiperglucémicos.

\section{Material y métodos}

\section{Plantas}

La $B$. veronicaefolia se recolectó en los alrededores de Taxco, Guerrero. En las cercanías de Tornacuxtla, Estado de México, se colectó la B. terniflora, y la P. edulis, en El Naranjo, estado de Veracruz. La bióloga Edith López Villafranco, del Departamento de Botánica de la Escuela Nacional de Estudios Profesionales-Iztacala, de la Universidad Nacional Autónoma de México, tuvo a su cargo la identificación de las plantas. Un espécimen de cada una se depositó en el herbario de dicha institución, con los registros 4256, 2765 y 5231, respectivamente.

\section{Animales}

Se utilizaron 336 ratones albinos (Mus musculus), cepa CD1, maduros sexualmente, de doce semanas de edad, en condiciones gonadales normales de intercelo, de ambos sexos, con un peso promedio de $35.0 \pm 5.0 \mathrm{~g}$ para los machos y de $30.0 \pm 5.0 \mathrm{~g}$ para las hembras. El bioterio de la Universidad Autónoma Metropolitana-Xochimilco proporcionó los animales sanos, mismos que se mantuvieron en un cuarto cerrado y aislado, en condiciones ambientales controladas de luz blanca. Se establecieron ciclos de luz-oscuridad de 12-12 horas de acuerdo con lo propuesto por Hafez. ${ }^{3}$ La luz se encendía a las 7:00 horas y se apagaba a las 19:00 horas. La temperatura ambiental se controló por medio del encendido y apagado del sistema de aire acondicionado, ${ }^{4}$ y fue de $23 \pm 1.0^{\circ} \mathrm{C}$; la humedad relativa fue de $45 \pm 5.0 \%$, medida con un barómetro marca Penn. El número de ratones que se utilizó para la investigación se dividió en 42 lotes de ocho animales cada uno. Se alimentaron con producto Purina y se les proporcionó agua ad-libitum.

\section{Preparación de los extractos}

Las hojas de B. veronicaefolia y B. terniflora, así como los frutos de P. edulis cortados en trozos pequeños, se extendieron sobre las mesas del laboratorio, evitando en lo posible la acción directa de los rayos solares. Una vez disecados al aire libre, ${ }^{5}$ las hojas y los frutos se molieron en un molino de bolas.

Durante cinco horas, $250 \mathrm{~g}$ de $B$. veronicaefolia, $B$. terniflora y P. edulis se trataron con 1.51 de hexano a temperatura de reflujo. Los extractos de cloroformo y metanol se prepararon con el mismo procedimiento. Después, los extractos se filtraron y finalmente se evaporaron a sequedad en un evaporador rotatorio a presión reducida, de tal modo que se obtuvo en cada caso un residuo verde con un rendimiento de $14 \%$ para $B$. veronicaefolia, $11 \%$ para B. terniflora y $8 \%$ en el caso de P. edulis. A partir de cada extracto se preparó una suspensión acuosa $(200 \mathrm{mg} / \mathrm{ml})$ que se utilizó para determinar la actividad antidiabética del mismo en los animales de experimentación.

\section{Bioensayo para la actividad hipoglucemiante}

\section{Estudios en ratones hiperglucémicos}

Se utilizaron ratones macho cepa CD1, a los cuales se inyectó una vez cada tercer día, hasta completar tres administraciones, una solución de aloxana $(70 \mathrm{mg} / \mathrm{kg}$ de peso del animal) y se les dejó descansar una semana. ${ }^{6}$ Se determinó el nivel de glucosa en sangre empleando el micrométodo de 0 -toluidina. ${ }^{7}$ Los animales que presentaron glucosa sanguínea superior a $150 \mathrm{mg} /$ $100 \mathrm{ml}$ se incluyeron en el grupo de animales diabé- 
ticos; en ratones normales en ayunas la concentración de glucosa en la sangre es de $80-110 \mathrm{mg} / 100 \mathrm{ml}$. Se usaron lotes de ocho animales cada uno; a estos últimos se les retiró el alimento 24 horas antes del experimento y se les proporcionó agua ad-libitum. Las suspensiones acuosas de los extractos se administraron por vía intraperitoneal (IP) a diferentes concentraciones. Al mismo tiempo, a los lotes control se les administró solución salina. El porcentaje de variación de glucemia se calculó de acuerdo con la siguiente fórmula:

$\%$ de la variación de glucemia $=\quad \frac{\text { Gx-Go }}{\text { Go }} \times 100$

$\mathrm{Go}=$ nivel inicial de glucemia

$\mathrm{Gx}=$ niveles de glucemia a las: 1.5, 3, 4.5 y 24 horas posteriores a la administración de extractos.

\section{Estudios en ratones normoglucémicos}

La evaluación de la actividad hipoglucemiante también se llevó a cabo mediante la determinación de la variación de los niveles de glucosa sanguínea en ratones normales después de la administración de los extractos de las plantas bajo estudio por vía IP. Se extrajeron muestras de sangre de la vena caudal por medio de un pequeño corte a las 1.5, 3, 4.5 y 24 horas posteriores a la administración del extracto. ${ }^{8}$

\section{Análisis estadístico}

A partir de los datos que se obtuvieron del estudio farmacológico sobre la actividad hipoglucemiante de $B$. veronicaefolia, $B$. terniflora y $P$. edulis, se determinó el análisis de varianza a un nivel de confianza de $95 \%$ y, cuando fue necesario, se compararon las medias aplicando la prueba de Tukey mediante el programa para computadora Microcal Origen.

\section{Resultados}

Los extractos clorofórmicos de B. terniflora y P. edulis presentaron actividad hipoglucemiante en ratones diabéticos, en comparación con el grupo control; el efecto de estos extractos se comparó, asimismo, con el efecto producido por la tolbutamida. Al administrar dosis de 100 a $300 \mathrm{mg} / \mathrm{kg} / \mathrm{IP}$ de los extractos de cloroformo de $B$. terniflora y $P$. edulis, se obtuvo una respuesta dosisdependiente; los resultados se presentan en el cuadro I. Los extractos metanólico y hexánico de estas plantas no presentaron actividad hipoglucemiante. El efecto hipoglucémico del extracto hexánico de B. veronicaefolia en ratones diabéticos también se presenta en el cuadro
I. Los extractos clorofórmico y metanólico de B. veronicaefolia no presentaron actividad hipoglucemiante. El efecto antidiabético de los extractos clorofórmicos de B. terniflora y P. edulis, y de hexánico de B. veronicaefolia a dosis de 100 a $300 \mathrm{mg} / \mathrm{kg} / \mathrm{IP}$ en ratones normoglucémicos se presentan en el cuadro II. Los resultados muestran que la solución salina usada como control en estos experimentos no produce cambios significativos en los niveles de glucosa sanguínea en animales tanto normales como hiperglucémicos.

\section{Discusión}

Con la administración del extracto clorofórmico de B. terniflora en ratones diabéticos la máxima actividad hipoglucemiante (58.56\% de reducción) se observó 4.5 horas después de la administración del extracto. Ese efecto persistía aun a las 24 horas, lo cual indica que la actividad hipoglucemiante de esta planta es de efecto similar al de la tolbutamida. Con la administración de $300 \mathrm{mg} / \mathrm{kg}$ del extracto hexánico de B. veronicaefolia en ratones diabéticos, la máxima actividad hipoglucemiante (72.13\% de reducción) se observó 4.5 horas después de la administración. La mínima actividad se presentó con la administración de $100 \mathrm{mg} / \mathrm{kg}$ (45.6\% de reducción), después de 4.5 horas de la administración. Este extracto presentó una respuesta dosis-dependiente, y el efecto persistió por un lapso menor a las 24 horas. Con la administración de $300 \mathrm{mg} / \mathrm{kg}$ del extracto clorofórmico de P. edulis, la máxima actividad hipoglucemiante (43.75\% de reducción) se observó 4.5 horas después de la administración. La mínima actividad se observó con la administración de $100 \mathrm{mg} / \mathrm{kg}$ (10.9\% de reducción), 1.5 horas después de la administración; ese efecto persistió a las 24 horas, lo cual indica que la actividad hipoglucemiante de esta planta es de efecto similar al de la tolbutamida.

Después de 4.5 horas de haberse administrado los extractos clorofórmicos de B. terniflora y P. edulis a dosis de $300 \mathrm{mg} / \mathrm{kg}$ en ratones normoglucémicos, se produjo la máxima actividad antidiabética (33.4 y $29.6 \%$ de reducción, respectivamente). El efecto persistió por un lapso superior a las 24 horas. Con el extracto hexánico de $B$. veronicaefolia en ratones normoglucémicos se observó una disminución estadísticamente significativa $(p<0.01)$ a las $1.5,3$ y 4.5 horas después de la administración; este efecto fue más pronunciado a las 4.5 horas (39.8\% de reducción), pero la concentración de glucosa se incrementa dentro de las 24 horas y los niveles de glucosa sanguínea regresan a los valores iniciales a las 24 horas, lo cual indica que el efecto de la $B$. veronicaefolia es de corta duración. 


\section{Cuadro I \\ Efecto de la administración de los extractos de B. terniflora, B. veronicaefolia y P. edulis a ratones con HIPERgLUCEMiA. México, 1996}

Estudio

Dosis $(\mathrm{mg} / \mathrm{kg})$

Porcentaje de variación de glucemia $(M \pm D E)$

\begin{tabular}{|c|c|c|c|c|c|c|}
\hline Control & & $328 \pm 1.41$ & $-0.30 \pm 1.43$ & $+0.40 \pm 1.50$ & $+0.38 \pm 1.40$ & $+0.76 \pm 1.59$ \\
\hline \multirow[t]{3}{*}{ B. terniflora ${ }^{a}$} & 100 & $239 \pm 3.60$ & $-15.89 \pm 0.78 *$ & $-24.55 \pm 0.92 *$ & $-34.89 \pm 1.66^{*}$ & $-6.54 \pm 3.97^{\ddagger}$ \\
\hline & 200 & $332 \pm 5.48$ & $-19.76 \pm 4.0^{*}$ & $-26.11 \pm 3.0^{*}$ & $-45.81 \pm 0.94 *$ & $-7.49 \pm 1.32 *$ \\
\hline & 300 & $287 \pm 4.60$ & $-23.11 \pm 0.77^{*}$ & $-35.02 \pm 1.32 *$ & $-58.56 \pm 0.88 *$ & $-8.72 \pm 1.21 *$ \\
\hline \multirow[t]{3}{*}{ B. veronicaefolia ${ }^{b}$} & 100 & $240 \pm 2.31$ & $-25.32 \pm 3.98 *$ & $-34.67 \pm 2.0 *$ & $-45.67 \pm 2.62 *$ & $+4.12 \pm 2.43^{*}$ \\
\hline & 200 & $270 \pm 3.27$ & $-35.16 \pm 2.43^{\ddagger}$ & $-46.71 \pm 3.4^{\ddagger}$ & $-67 \pm 4.25^{\ddagger}$ & $+3.44 \pm 0.87 *$ \\
\hline & 300 & $301 \pm 4.46$ & $-43.34 \pm 3.31 *$ & $-56.05 \pm 1.67^{*}$ & $-72.13 \pm 2.97 *$ & $+1.89 \pm 2.08^{*}$ \\
\hline \multirow[t]{3}{*}{ P. edulis ${ }^{\mathrm{a}}$} & 100 & $225 \pm 1.98$ & $-10.90 \pm 0.92 *$ & $-18.71 \pm 1.68 *$ & $-27.16 \pm 3.67^{\ddagger}$ & $-4.38 \pm 3.52^{\ddagger}$ \\
\hline & 200 & $265 \pm 2.84$ & $-14.83 \pm 1.74 *$ & $-26.21 \pm 4.03^{\ddagger}$ & $-39.5 \pm 3.16^{\ddagger}$ & $-6.78 \pm 0.79 *$ \\
\hline & 300 & $311 \pm 1.36$ & $-18.41 \pm 2.80^{\ddagger}$ & $-29.64 \pm 3.06^{\ddagger}$ & $-43.75 \pm 1.32 *$ & $-8.05 \pm 1.37^{*}$ \\
\hline Tolbutamide & 50 & $310 \pm 2.06$ & $-24.7 \pm 1.64 *$ & $-35.1 \pm 3.54^{\ddagger}$ & $-63.1 \pm 1.39 *$ & $-9.72 \pm 1.21 *$ \\
\hline
\end{tabular}

M: media

DE: desviación estándar

Control: vehículo. Extractos: cloroformo, ${ }^{a}$ hexano. ${ }^{b}$ Los valores finales fueron comparados con los iniciales. Los niveles de glucosa sanguínea disminuyen significativamente en los grupos tratados e incrementan en el grupo control

$* p<0.001$

$\neq p<0.01$

\section{Cuadro II \\ Efecto de la administración de los extractos de B. terniflora, B. veronicaefolia y P. edulis a ratones NORMOGLUCÉMICOS. MÉXICO, 1996}

Estudio

Dosis $(\mathrm{mg} / \mathrm{kg})$

Porcentaje de variación de glucemia $(M \pm D E)$

\begin{tabular}{|c|c|c|c|c|c|c|}
\hline Control & & $105 \pm 2.08$ & $-0.01 \pm 1.32$ & $+0.01 \pm 2.36$ & $+0.01 \pm 2.27$ & $-0.03 \pm 3.18$ \\
\hline \multirow[t]{3}{*}{ B. terniflora ${ }^{a}$} & 100 & $110 \pm 1.41$ & $-5.71 \pm 2.20^{\ddagger}$ & $-14.3 \pm 1.71 *$ & $-26.7 \pm 1.16 *$ & $-3.8 \pm 3.02^{\ddagger}$ \\
\hline & 200 & $103 \pm 1.40$ & $-7.38 \pm 3.51^{\ddagger}$ & $-16.43 \pm 2.67 *$ & $-28.23 \pm 1.06^{*}$ & $-8.1 \pm 3.29^{\ddagger}$ \\
\hline & 300 & $100 \pm 3.34$ & $-8.23 \pm 1.06 *$ & $-25.13 \pm 3.63^{\ddagger}$ & $-33.42 \pm 4.53^{\ddagger}$ & $-9.10 \pm 2.31 *$ \\
\hline \multirow[t]{3}{*}{ B. veronicaefoliab } & 100 & $104 \pm 0.98$ & $-7.32 \pm 0.98 *$ & $-17.72 \pm 1.05 *$ & $28.01 \pm 0.87 *$ & $+1.39 \pm 1.68 *$ \\
\hline & 200 & $101 \pm 2.16$ & $-9.06 \pm 2.67^{\ddagger}$ & $-19.43 \pm 4.25^{\ddagger}$ & $-31.07 \pm 3.54^{\ddagger}$ & $+1.05 \pm 4.11^{\ddagger}$ \\
\hline & 300 & $106 \pm 1.54$ & $-12.35 \pm 3.76^{\ddagger}$ & $-32.16 \pm 2.74^{*}$ & $-39.84 \pm 2.33^{*}$ & $+0.82 \pm 3.41^{\ddagger}$ \\
\hline
\end{tabular}

\begin{tabular}{lclllll} 
P. edulis $^{\mathrm{a}}$ & 100 & $105 \pm 1.79$ & $-4.31 \pm 0.85^{*}$ & $-12.56 \pm 3.56^{\ddagger}$ & $-22.43 \pm 2.60^{*}$ & $-2.89 \pm 2.64^{*}$ \\
\hline & 200 & $107 \pm 3.20$ & $-6.75 \pm 3.25^{\ddagger}$ & $-14.89 \pm 1.76^{*}$ & $-26.73 \pm 1.33^{*}$ & $-6.56 \pm 1.49^{*}$ \\
\hline Tolbutamide & 300 & $102 \pm 2.08$ & $-7.03 \pm 1.59^{*}$ & $-20.61 \pm 1.80^{*}$ & $-29.61 \pm 2.25^{*}$ & $-7.86 \pm 2.19^{\ddagger}$ \\
\hline 50 & $101 \pm 1.67$ & $-8.13 \pm 3.29^{\ddagger}$ & $-24.10 \pm 2.79^{*}$ & $-31.05 \pm 3.32^{\ddagger}$ & $-8.76 \pm 3.0^{*}$
\end{tabular}

M: media

DE: desviación estándar

Control: vehículo. Extractos: cloroformo, ${ }^{a}$ hexano. ${ }^{b}$ Los valores finales fueron comparados con los iniciales. Los niveles de glucosa sanguínea disminuyen significativamente en los grupos tratados e incrementan en el grupo control

$* p<0.001$

$\neq p<0.01$ 


\section{Conclusiones}

Las plantas B. veronicaefolia, B. terniflora y P. edulis utilizadas en la medicina tradicional para el tratamiento de la diabetes presentaron actividad hipoglucémica en ratones normales y diabéticos.

\section{Referencias}

1. Bates RB, C ole JR. Solution form of Bouvardin and relatives from N MR studies. J Am Chem Soc 1983;32:1343-1347.
2. Perez-G RM. Effect of an aqueous extract of Brickellia veronicaefolia on the gastrointestinal tract of Guinea-pig, rats and mice. Phytother Res 1996;10(6):677-679.

3. Hafez EC. Reproduction and breeding. Techniques for laboratory animals. Filadelfia: Ed. E.S.E. H afez Phil. Lea \& Fehiger, 1970:126-135.

4. Turner R. Screening methods in pharmacology. Filadelfia: Academic Press, 1965:243-281.

5. Casamada RSM. Tratado de farmacognosia. Barcelona: CientíficoMédica, 1977:26-28

6. Rodríguez GH, Pérez-G RM, Muñoz MH, Pérez GC. Inducción de diabetes en ratón por medio de aloxana. Acta Medica 1975;6:33-36.

7. Hyavariner A. N ikkita E. Specific determination of blood glucose with 0-toluidine. Clin Chim Acta 1966;7:140-143.

8. Dulin W.E. Basic pharmacological techniques for evaluating antidiabetic agent. Chicago:Year Book Medical Publishers, 1964:210-250. 